https://doi.org/10.7203/Normas.1.4651

\title{
LA ENSEÑANZA DEL ESPAÑOL A LOS HISPANOHABLANTES DE HERENCIA DE LOS ESTADOS UNIDOS
}

\author{
TEACHING SPANISH TO HERITAGE SPANISH SPEAKERS FROM THE UNITED \\ STATES
}

\author{
Antonio TORRES TORRES \\ Universitat de Barcelona
}

\section{RESUMEN:}

En este artículo se analiza el tipo de español que se enseña en los cursos a los hispanohablantes de herencia de los Estados Unidos. Con el crecimiento de la población de origen hispánico en aquel país se ha incrementado el número de estudiantes de español que lo tienen como lengua patrimonial. Por este motivo, se han desarrollado en las últimas décadas investigaciones sobre cómo debe ser la enseñanza del español a este tipo de alumnos, se han elaborado materiales docentes y se han puesto en marcha cursos específicos de Español para Hablantes Nativos. Habitualmente, se considera necesario que los hispanohablantes incorporen una forma estándar de español que se sume a las variedades populares y coloquiales que traen consigo, de modo que puedan usar un registro adecuado en cada situación.

PALABRAS ClAVE: enseñanza del español, hispanohablantes de herencia, Estados Unidos, variedades del español.

\section{ABSTRACT:}

This article discusses the type of Spanish taught in the courses aimed at heritage Spanish speakers from the United States. With the growth of Hispanic population in that country, the number of students of Spanish who have it as a heritage language has increased. For that reason, in recent decades, research on teaching Spanish to those students has been developing, resources have been created, and specific courses for native Spanish speakers have been established. Usually, it is considered to be necessary that Spanish speakers incorporate a standard form of Spanish which adds to the popular and colloquial varieties they bring with them, so they can use a register adequate to each situation.

KEY WORDS: teaching Spanish, heritage Spanish speakers, United States, varieties of Spanish. 


\section{INTRODUCCIÓN}

Aun contando con la presencia histórica del español en el territorio de los actuales Estados Unidos, ha sido a lo largo del siglo XX y de lo que llevamos del XXI cuando se ha producido un notable incremento, especialmente en las últimas décadas, de las cifras de población de origen hispanohablante en el país, así como de las variedades de español que maneja, en función de su origen geográfico y sociocultural, y también de los distintos grados de contacto con el inglés. Según los datos correspondientes al censo de 2010, hay 50,5 millones de hispánicos en los Estados Unidos, lo que representa el $16,3 \%$ de la población total. Este grupo ha crecido un $43 \%$ durante la última década (estaba formado por 35,3 millones en el año 2000) y supone el 56\% del aumento total de la población, esto es, más de la mitad del crecimiento demográfico de los Estados Unidos corresponde a los hispánicos. La mayor parte de ellos (un 76\%) vive, en 2010, en nueve estados: Arizona, California, Colorado, Florida, Illinois, Nuevo México, Nueva Jersey, Nueva York y Texas, pero ese porcentaje refleja un descenso sostenido ( $81 \%$ en 2000 y $86 \%$ en 1990), de lo que se sigue que se produce una mayor dispersión hispánica por el territorio de la Unión (Pew Hispanic Center).

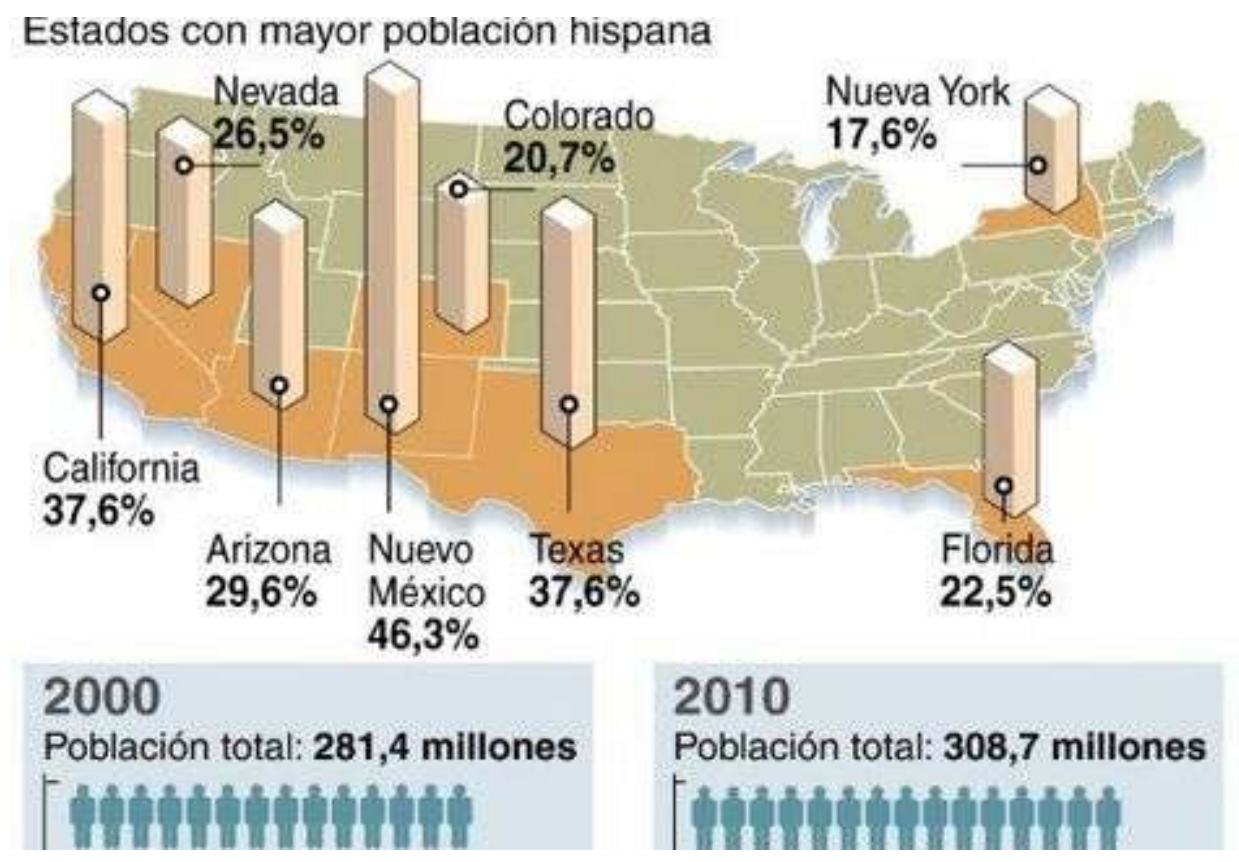

Figura 1. Estados con mayor porcentaje de población hispana de los Estados Unidos. Fuente: <http://www.prensalibre.com/internacionales/Mapa-hispanos-Unidos_PREIMA20110324_0261_5.jpg> 


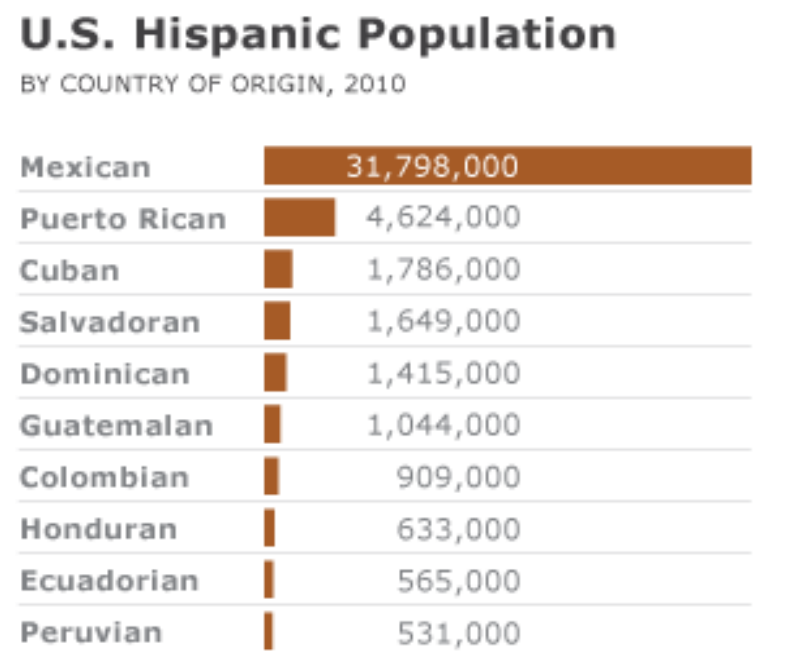

Figura 2. Población hispánica de los Estados Unidos, según los diez primeros países de origen, en 2010

Fuente: Pew Hispanic Center

Las cuestiones candentes en el análisis del español en los Estados Unidos son, por un lado, la perspectiva dialectológica que permita delimitar las distintas variedades con una base territorial, los aspectos que derivan del contacto entre el inglés y el español, con el debate sobre la naturaleza del llamado spanglish (Torres 2001, 2005, 2010a y $2010 b)$ y, por otro, la enseñanza del español a los hablantes de herencia latinos, así como la discusión sobre el modelo de español elegido con fines docentes.

\section{FACTORES QUE PUEDEN DESINCENTIVAR EL ESTUDIO DEL ESPAÑOL EN LOS ESTADOS UNIDOS}

A pesar de la mayor visibilidad pública de los latinos en los Estados Unidos, día a día se ven sometidos a numerosas presiones asimilacionistas, como ilustran las andanadas contra los inmigrantes de ese origen que en los últimos tiempos lanzó el profesor Samuel Huntington, las proclamas que enarbolan grupos como U.S. English 〈www.us-english.org> o English Only 〈www.us-english.org/inc〉, así como las actuaciones contra la educación bilingüe (recuérdese la Proposición 227, aprobada en California en 1998, con el voto favorable del $61 \%$ de los californianos, a la que han seguido medidas similares en otros estados, como Arizona, en 2000, y Massachusetts, en 2002; se han sucedido las leyes para afianzar en el inglés su carácter de «lengua de los Estados Unidos») ${ }^{1}$ y contra la affirmative action, que desembocan en la intolerancia

\footnotetext{
${ }^{1}$ Ofelia García (2008a) resume los avatares por los que ha pasado la educación bilingüe en los Estados Unidos.
} 
lingüística y en una imagen negativa de los hispánicos y del español en los Estados Unidos.

En la política lingüística que se aplica en el país no se favorece que los niños que no hablan inglés mantengan su lengua nativa a la par que aprenden la nueva, sino que adquieran esta última por encima de todo, a pesar de la paradoja que encierra tal planteamiento, como ha hecho notar Kim Potowski (2005: 23):

[...] nos parece una política completamente ilógica el 'eliminar' el español [...] de los niños latinos durante sus años de escolarización, para después exigirles que lo estudien como idioma extranjero cuando lleguen a la escuela secundaria. Tendría mucho más sentido permitirles que lo sigan desarrollando durante los años tempranos.

Con lo anterior concuerda la atención limitada que se ha prestado en los Estados Unidos a la enseñanza de lenguas extranjeras. En concreto, no son demasiados los programas de español como LE en las escuelas primarias (solo un 31\% de ellas imparten lengua extranjera) y, por lo que respecta a las escuelas secundarias y a las universidades, donde se concentra la docencia del español, se ha señalado el reducido número de horas consagradas a dicha tarea, y la preparación insuficiente de algunos profesores. De hecho, como afirma Ofelia García (2008b: 427), «[e]l español se enseña en los Estados Unidos a través del inglés». Por todo ello, criar a un niño bilingüe en aquel país puede ser considerado un acto político (Ana Celia Zentella et alii 1998: 9).

\section{FACTORES QUE PUEDEN INCENTIVAR EL ESTUDIO DEL ESPAÑOL EN LOS ESTADOS UNIDOS}

Del estudio del español, además del inglés, en los Estados Unidos, derivan beneficios de orden personal, cultural, académico y profesional. En primer lugar, los especialistas resaltan las ventajas cognitivas del bilingüismo (Roca y Colombi 20032006); en el aprendizaje formal del español se pueden aprovechar las destrezas desarrolladas antes en inglés. Además, el bilingüismo conlleva ventajas para toda la sociedad.

La motivación para aprender una segunda lengua, y para reforzar el papel del español en los latinos, también presenta un cariz económico: de la misma manera que manejar correctamente el inglés permite la integración en la sociedad mayoritaria de los Estados Unidos, dominar el español abre las puertas a un mercado global. Esto lo prueba la creciente demanda del español como lengua extranjera en la Unión. Es la lengua más popular en la enseñanza secundaria: «En los grados 7 a 12, un $70 \%$ estudia español, mientras que solo un $18 \%$ selecciona el francés, un $5 \%$ el alemán, un $3 \%$ el 
latín y un 1\% el italiano» (García 2008b: 425). Por su parte, Kim Potowski y Andrew Lynch (2004: 24) han mostrado que en el ámbito universitario estadounidense en 1968 estudiaba español el 32,4\% de los alumnos matriculados en LE; en 1980 el porcentaje ascendió al 41\%; en 1990, al 45,1\%; y en 1998, al 55\% (a pesar de ello, las cifras son más bajas que las que corresponden a la escuela secundaria). En 2006, el número total de matriculados en español en los distintos niveles universitarios era de 822 985; en francés, de 206 426; y, en alemán, de 94264 (Domínguez 2008: 439). Además, hay que tener en cuenta la acción educativa del Ministerio de Educación y Ciencia español en los Estados Unidos, la presencia en el país del Instituto Cervantes a través de cuatro sedes (Nueva York, Chicago, Albuquerque y Seattle), de distintas instituciones hispanoamericanas, sobre todo de México, y de algunos centros privados (cf. Domínguez 2008: 437-446).

En el caso específico de los latinos, el estudio del español está muy vinculado, asimismo, a cuestiones étnicas, al refuerzo de una identidad, a los lazos compartidos con el resto del mundo hispánico, aunque muchas veces el espacio intercultural en el que se mueven y el desarrollo de variedades lingüísticas híbridas en la órbita del denominado spanglish puedan difuminar ese encaje siempre difícil. De todos estos provechos también es necesario concienciar a los padres. En este sentido tiene mucha utilidad el folleto «Would you like your children to speak English and Spanish?» / «¿Quieren que sus hijos hablen el inglés y el español?», dirigido por Ana Celia Zentella (1998), en apoyo del bilingüismo inglés-español en los niños latinos.

\section{LOS CURSOS PARA LOS HABLANTES DE HERENCIA Y LA ENSEÑANZA DE UNA VARIEDAD ESTÁNDAR DE ESPAÑOL}

Aquellos alumnos latinos de los Estados Unidos que ya poseen, en una u otra medida, conocimientos nativos del español, esto es, que lo tienen como lengua «patrimonial», son llamados «hablantes bilingües», «hablantes nativos», «hispanohablantes»o «hablantes de herencia» (heritage speakers) ${ }^{2}$. Sus características son muy variadas. Hallamos estudiantes cuyas familias tienen un arraigo de varias generaciones en los Estados Unidos, y en los que el inglés es la lengua dominante, frente a un conocimiento elemental del español, un bilingüismo pasivo o receptivo que únicamente les permite cierta comprensión de esta lengua. Otros, principalmente de segunda generación, que han recibido su educación formal en inglés, una lengua que

${ }^{2}$ Ofelia García critica la etiqueta reciente de «español como lengua de herencia», que «deja de nombrar una realidad estadounidense: el hecho de que somos muchos en el país los Spanish speakers. Además, el concepto de 'lengua de herencia' encubre al español por el inglés, para muchos 'la única lengua que cuenta, la única moderna y del presente, la única del futuro’. Para muchos de los que hablamos español en los Estados Unidos, este idioma no es una herencia, es una de las lenguas de nuestro presente y de nuestro futuro» (García 2008b: 425).

NORMAS. REVISTA DE ESTUDIOS LINGÜÍSTICOS HISPÁNICOS, NÚMERO 1 (AÑO 2011): http://www.uv.es/normas 
manejan con soltura, y que utilizan el español con bastante fluidez, normalmente en situaciones informales, pero que no manejan en su registro académico. Asimismo, podemos encontrar inmigrantes recientes en quienes el español es la lengua dominante.

En el terreno de la enseñanza, mezclar en una clase a estos estudiantes con los que parten directamente del inglés en su aproximación al español resulta complicado, no solo para los dos tipos de aprendientes, sino también para el profesor. Como señala Kim Potowski (2005: 84),

[1]os alumnos anglohablantes pueden sentirse intimidados por las habilidades orales en español de los hispanos; los hispanos se pueden sentir intimidados por las habilidades gramaticales de sus compañeros anglohablantes; el instructor muchas veces no sabe cómo dividir su atención para cumplir con las necesidades diferentes de los dos grupos.

A pesar de todo, y aunque desde los años setenta del siglo pasado se trabaja, en los Estados Unidos, con el fin de lograr cursos específicos para estos alumnos Guadalupe Valdés fue la iniciadora de este campo-, existen pocos programas de español para nativos: solo el $18 \%$ de las universidades del país (según datos de un estudio de 2002) y el 9\% de escuelas de secundaria (según datos de 1997) los ofrecía. Así, «la gran mayoría de los estudiantes hispanohablantes del país reciben instrucción en su lengua nativa como si fuera para ellos una lengua extranjera» (Potowski y Lynch 2004: 36), lo que resulta inadecuado. Los cursos que más se ajustan a ellos son los de Español para Hablantes Nativos (EHN) o Spanish for Native Speakers (SNS) en inglés, que parten específicamente de la perspectiva del hablante nativo, por muy diversos que sean sus niveles en el manejo del español, en relación con numerosos factores, como la variedad geográfica y social usada por la familia, la instrucción formal recibida en español o la generación a la que pertenecen (en función de la cual estarán más o menos presentes en su expresión lingüística los fenómenos de contacto entre el español y el inglés: préstamos, calcos, mezcla de códigos, cambio de códigos).

En general, los hablantes de herencia muestran un notable desajuste entre las destrezas lingüísticas. Al principio puede sorprender al profesor una producción oral prácticamente asimilable a la de un nativo, y una capacidad elevada de comprensión del español, pero pronto se dará cuenta de que el estudiante de ascendencia hispánica manifiesta carencias en determinados campos léxicos y una falta de conocimiento explícito de la gramática del español, así como dificultades de lectura y de escritura. Además, es frecuente que los latinos, a pesar de la naturalidad con que manejan el español, obtengan calificaciones inferiores a las de sus compañeros anglosajones, que muestran un progreso constante y equilibrado en el aprendizaje de la lengua.

Por lo que se refiere concretamente al diseño de los cursos de EHN, se propone, por un lado, que deberían atender a las necesidades socioafectivas de los estudiantes, para eliminar sus miedos e inseguridades en el manejo del español, para fortalecer los vínculos con la comunidad hispánica, y para aumentar su autoestima, a lo que ayuda la 
presentación de figuras hispánicas relevantes en la historia y el presente de los Estados Unidos, como escritores (en español), directores de cine, músicos, etc. Por otro lado, estos cursos deben tener como objetivo la adquisición por parte del estudiante de una variedad estándar de español que no sustituya su dialecto propio, local, coloquial, sino que se añada a él: se debe promover el «bidialectismo» aditivo. Como ha señalado Marta Fairclough (2003: 7), «una pedagogía bidialectal parece ser el camino más indicado a seguir por aquellos que tienen como tarea la enseñanza del español estándar al estudiantado que compone los cursos de español para hispanoparlantes bilingües en los Estados Unidos».

Para ello es necesario fomentar en los alumnos la conciencia lingüística que les permita separar de modo sistemático la variedad popular-dialectal propia de la estándar o de la norma culta que se tome como modelo. Los análisis contrastivos constituyen un ejercicio fundamental ${ }^{3}$. Como escribe Kim Potowski (2005: 40-41),

[...] muchos chicos bilingües llegan al aula con un español de playa y nuestra meta es ampliarles el vestuario para que puedan vestirse apropiadamente para los eventos más formales. Es decir, queremos ayudarles a expandir y pulir el español que ya traen consigo. La meta nunca debe ser eliminar el español que tienen, por mucha influencia del inglés o rasgos estigmatizados que lo caractericen, ya que les pertenece a ellos y a sus familias, y lo van a necesitar siempre que se encuentren en esos contextos.

En estos cursos, por tanto, se deberían describir y analizar los fenómenos de contacto entre el español y el inglés que les son propios, sin ningún tipo de condena, y se tendrían que desplegar en los alumnos las destrezas comunicativas y, en particular, las que atañen a los contextos académicos y profesionales, lo que supone desarrollar también las habilidades de lectura y escritura, y tener en cuenta la inserción de estos hablantes en una estructura social, pública. Como escribe María Cecilia Colombi (2001: punto 2),

[e]l desarrollo del lenguaje académico va más allá de saber leer y escribir; es necesario aprender a usar el lenguaje en formas que demandan un conocimiento específico del lenguaje, de los textos y de los nuevos instrumentos tecnológicos para convertirse en participantes activos en nuestra sociedad actual.

El reto para los profesores es facilitar esa alfabetización avanzada.

No escapa a la reflexión sobre la pedagogía «bidialectal» el propio concepto de estándar y la multiplicidad a través de la que se manifiesta la norma culta. De acuerdo con Francisco Moreno Fernández (2010: 99), «[1]a estandarización del español es de

${ }^{3}$ En el apéndice se incluyen varios textos producidos por estudiantes latinos que han seguido mis cursos en la Universidad de Barcelona, y que posibilitan la realización de esa labor.

NORMAS. REVISTA DE ESTUDIOS LINGÜÍSTICOS HISPÁNICOS, NÚMERO 1 (AÑO 2011):

http://www.uv.es/normas

(ISSN 2174-7245) 
naturaleza "monocéntrica" (norma académica única) construida sobre una realidad polinormativa (norma culta policéntrica)». De este modo, existe un español estándar sobre una pluralidad de normas cultas. A la vez, la política lingüística académica aboga por el panhispanismo, esto es, por «consensuar las normas de corrección de la lengua, favoreciendo lo común y sacrificando lo más particular» (Moreno Fernández 2010: 100). En el extremo del panhispanismo se situaría una «reestandarización» del español, una nueva modalidad de la lengua, creada artificialmente para ser usada sobre todo en los medios de comunicación y en la enseñanza, que se conoce como español internacional, español neutro, español globalizado, etc.

Se considera que los hablantes de herencia latinos deben ser conscientes de la variación del español y de los factores que inciden sobre esa diversidad. Al mismo tiempo, en la enseñanza del español a estos estudiantes se debe tomar como referencia una norma culta:

Esa norma culta, en el caso de los Estados Unidos, bien puede ser la mexicana, para todo el Suroeste, o la caribeña, para Florida y el Nordeste, siempre con la aceptación de los fenómenos derivados del contacto con el inglés que más se han generalizado en el uso del español (Moreno Fernández 2010: 152).

Cynthia Ducar (2009) parte de que el objetivo central en las clases de español para hablantes de herencia y el tema más tratado en la investigación al respecto se cifra en la adquisición de un segundo dialecto (un dialecto estándar), pero indica que no ha habido hasta la fecha estudios críticos sobre cómo se abordan los dialectos del español estadounidense en libros de texto para hablantes de herencia. Así, a partir del método para el análisis crítico del discurso que plantea Teun A. van Dijk, investiga el tratamiento de la lengua en cuatro manuales universitarios de nivel intermedio para hablantes de herencia: Español escrito, La lengua que heredamos, Mundo 21 hispano y Nuevos mundos. Concluye que estos libros presentan el español de los Estados Unidos como problemático, silencian sus características y apelan a un estándar pseudopeninsular, y realiza varias sugerencias pedagógicas, que persiguen la inclusión de investigación sociolingüística y de datos basados en corpus, así como de ejercicios de traducción bidireccional entre una forma estándar y variedades regionales del español, con lo que se confiere valor a estas últimas.

Es evidente que los profesores de EHN necesitan una formación específica, pues no basta con dominar las estrategias aplicadas en la enseñanza de ELE, sino que deben saber cuáles son las singularidades de sus alumnos:

Así, deben conocer el contexto social de los hispanos, la situación del español, las variedades de español que se hablan en Estados Unidos y deben estar familiarizados con los conceptos sociolingüísticos fundamentales. Es importante que los profesores sean 
conscientes de las características lingüísticas y las experiencias culturales y académicas que cada estudiante lleva a clase (Moreno Fernández 2010: 180).

Por otro lado, a medida que se ha ido implantando este tipo de cursos y que se ha desarrollado la investigación sobre el tema, han ido apareciendo materiales diversos ${ }^{4}$, como los citados arriba, aunque todavía es necesario avanzar en este terreno. Sea como sea, a menudo existen dificultades de orden práctico que no permiten la puesta en marcha de cursos para los latinos, en los Estados Unidos al igual que en el exterior, como un número insuficiente de estudiantes para iniciarlos, una gran disparidad de niveles dentro del grupo que se podría formar, o las dinámicas académicas ya fijadas que se repiten año tras año.

\section{CONCLUSIÓN}

A lo largo de las últimas décadas, la población hispánica crece de forma intensa en los Estados Unidos, y las previsiones apuntan a que lo seguirá haciendo en el futuro. Esta circunstancia ha comportado el aumento de un tipo de estudiantes de español, los de origen hispanohablante, que no se pueden asimilar a los que tienen el inglés como primera lengua. Consecuentemente, también se ha hecho necesario reflexionar sobre las necesidades específicas de este grupo cada vez más nutrido de alumnos de español, y plantear la mejora del entorno de enseñanza de esta lengua a los hispanohablantes de herencia. Un aspecto esencial al que se debe atender es el de la conciliación en el aula de las distintas variedades de español que hablan los latinos con el desarrollo de una norma culta, encaminada hacia la variedad estándar de la lengua. Por un lado, los estudiantes deben percibir que las variedades de español que manejan tienen valor y no deben ser eliminadas; por otro, también deben ser conscientes de que existen muchas formas de usar la lengua (que cambian en función del territorio, del nivel sociocultural, de la situación, de la lengua con la que el español se halla en contacto en muchos lugares, y de otros factores), y de que es positivo y necesario dominar los recursos que la lengua brinda a los hablantes y tener la habilidad de utilizar un código estándar en determinados contextos.

\footnotetext{
${ }^{4}$ Cf. Suárez (2004: 820-822) para los aspectos abordados generalmente en los manuales: la cultura de los países hispánicos, las lecturas, la escritura (y, en particular, la ortografía), el vocabulario y las reglas gramaticales.
}

NORMAS. REVISTA DE ESTUDIOS LINGÜÍSTICOS HISPÁNICOS, NÚMERO 1 (AÑO 2011): http://www.uv.es/normas 


\section{RECURSOS Y REFERENCIAS BIBLIOGRAFICAS}

ABDUL-BAGI, Samia (2008): «La enseñanza del español como lengua de herencia en los Estados Unidos: impresiones de una pedagoga en Filadelfia», Consensus, ene./dic. 2008, vol.13, n. ${ }^{\circ}$ 1, 49-56. Disponible en Internet: $\langle$ http://revistas.concytec.gob.pe/pdf/consen/v13n1/a06v13n1.pdf $>$ [consulta: 18/07/2011].

Asociación de Profesores de Español y Portugués de los Estados Unidos (The American Association of Teachers of Spanish and Portuguese, AATSP). <http://www.aatsp.org/scriptcontent/index.cfm>.

BetTI, Silvia (2011): “"Yo quería cruzar la línea...” Migrazione, frontiera e identità. I latinos negli Stati Uniti», Confluenze. Rivista di studi iberoamericani, vol. 3, n. $^{\circ}$ 1, 107-125. <http://confluenze.cib.unibo.it/article/view/2203/1589> [consulta: 20/07/2011].

Callahan, Laura (2010): «U.S. Latinos’ Use of Written Spanish: Realities and Aspirations», Heritage Language Journal, 7(1), 1-27. $\langle$ http://www.international.ucla.edu/media/files/vol7-1-R-Callahan.pdf $>$ [consulta: $11 / 03 / 2011]$.

CARreIRA, Maria (2004): «Seeking Explanatory Adequacy: A Dual Approach to Understanding the Term Heritage Language Learner», Heritage Language Journal, vol. 2 n. ${ }^{\circ}$ $<$ http://www.international.ucla.edu/languages/heritagelanguages/journal/article.asp?par entid=14647> [Consulta: 18/07/2011].

CAStro, Max J. (2007): «The Future of Spanish in the United States», Our World, 13 Dec. 2007 <http://ourworld.compuserve.com/homepages/JWCRAWFORD/Castro1.htm > [consulta: 05/06/2010].

Centro Virtual Cervantes. Índice de recursos «El español en Estados Unidos». $<$ http://cvc.cervantes.es/obref/espanol_eeuu/indice.htm $>$.

Colombi, María Cecilia (2001): «El desarrollo del registro académico del español en estudiantes latinos en EE.UU.», en II Congreso Internacional de la Lengua Española, Valladolid, 2001, <http://congresosdelalengua.es/valladolid/ponencias/unidad_diversidad_del_espanol/3_ el_espanol_en_los_EEUU/colombi_m.htm> [consulta: 22/03/2011].

Colombi, María Cecilia y Francisco X. Alarcón (eds.) (1997): La enseñanza del español a hispanohablantes: Praxis y teoría, Boston, Houghton Mifflin. 
CRIADO, María Jesús (2004): «Escenarios y tendencias de la lengua española», en Vanguardia dossier. Los hispanos en Estados Unidos, n. ${ }^{\circ}$ 13, octubre/diciembre 2004, 87-93.

DomíngueZ, Carlos (2008): «La enseñanza del español en cifras», en Humberto López Morales, coord.: Enciclopedia del español en los Estados Unidos, Madrid, Instituto Cervantes/Santillana, 429-448.

DuCAR, Cynthia (2009): «The sound of silence: Spanish heritage textbooks' treatment of language variation», en Manel Lacorte y Jennifer Leeman, eds., Español en Estados Unidos y otros contextos de contacto. Sociolingüistica, ideología y pedagogía I Spanish in the United States and other contact environments. Sociolinguistics, ideology and pedagogy, Madrid, Iberoamericana/Frankfurt am Main, Vervuert, 347-367.

ESPINOZA MOORE, Jaime E. (2010): Los hispanohablantes de herencia en las escuelas secundarias: el caso práctico de Worthington, Ohio. (A Thesis Presented to The Honors Tutorial College Ohio University in Partial Fulfillment of the Requirements for Graduation from the Honors Tutorial College with the degree of Bachelor of Arts in Spanish). $<$ http://etd.ohiolink.edu/sendpdf.cgi/Espinoza\%20Moore\%20Jaime\%20E.pdf?ouhonors1275673327> [consulta: 18/06/2011].

FAIRCLOUGH, Marta (2003): «La adquisición de un segundo dialecto: el español estándar en una situación de contacto», 〈http://www.ub.es/ice/portaling/seminari/seminari-pdf/52fairclough.pdf $>$ [consulta: 23/03/2011]. (Publicado en Joan Perera, ed.: Plurilingüisme i educació: els reptes del segle XXI. Ensenyar llengües en la diversitat $i$ per a la diversitat, Barcelona, Institut de Ciències de l'Educació, Universitat de Barcelona, 365-372).

GARCÍA, Ofelia (2003): «La enseñanza del español a los latinos de los EE. UU.: contra el viento del olvido y la marea del inglés», Ínsula, n. ${ }^{\circ}$ 679-680, 9-12.

GARCÍA, Ofelia (2008a): «El uso del español en la enseñanza. La educación bilingüe», en Humberto López Morales, coord.: Enciclopedia del español en los Estados Unidos, Madrid, Instituto Cervantes/Santillana, 417-422.

GARCÍA, Ofelia (2008b): «La enseñanza del español como lengua extranjera», en Humberto López Morales, coord.: Enciclopedia del español en los Estados Unidos, Madrid, Instituto Cervantes/Santillana, 423-428.

Gineste LlOMBART, Albert (2006): «Latinos en EE.UU. (Desarrollando la competencia comunicativa intercultural)», Revista redELE (red electrónica de didáctica del Español como Lengua Extranjera), número 6, segundo semestre 2006. $<$ http://www.mec.es/redele/Biblioteca2006/AGineste.shtml $>$ [última consulta: 24/05/2011].

LLORENTE, Lucía I. (2004): «Los hispanohablantes en las clases regulares de español: ¿ventaja o desventaja?», en Manuel Pérez Gutiérrez y José Coloma Maestre, eds.: El español, lengua del mestizaje y la interculturalidad (Actas del XIII Congreso 
Internacional de la Asociación para la Enseñanza del Español como Lengua Extranjera, ASELE) (Murcia, 2-5 de octubre de 2002), 550-559. En Internet: $\langle$ http://www.mepsyd.es/redele/biblioteca/asele/49.1lorente_berry.pdf $>$ [consulta: 06/05/2011].

LÓPEZ Morales, Humberto, coord. (2008): Enciclopedia del español en los Estados Unidos, Madrid, Instituto Cervantes/Santillana.

Marcos-Marín, Francisco A. (2005): «Pluralidad del español en los Estados Unidos de América», en Centro Virtual Cervantes, Anuario 2005, $<$ http://cvc.cervantes.es/obref/anuario/anuario_05/marin/> [consulta: 22/01/2011].

MiKULSKI, Ariana M. (2010): «Age of Onset of Bilingualism, Language Use, and the Volitional Subjunctive in Heritage Learners of Spanish», Heritage Language Journal, 7(1), 28-46. <http://www.international.ucla.edu/media/files/vol7-1-AMIKULSKI.pdf> [consulta: 18/03/2011].

Montrul, Silvina y Melissa Bowles (2010): «Is Grammar Instruction Beneficial for Heritage Language Learners? Dative Case Marking in Spanish», Heritage Language Journal, 7(1), 47-73. <http://www.international.ucla.edu/media/files/vol7-1-S-MontrulM-Bowles.pdf> [consulta: 25/03/2011].

MoReno FERnÁNDEZ, Francisco (2010): Las variedades de la lengua española y su enseñanza, Madrid, Arco/Libros (Manuales de formación de profesores de español 2/L).

MraK, N. Ariana (2003): «La enseñanza del español estándar a los estudiantes hispanoparlantes de Estados Unidos: diseño de un curso universitario», $\langle$ http://www.ub.es/ice/portaling/seminari/seminari-pdf/54mrak.pdf $>$ [consulta: 23/03/2011]. (Publicado en Joan Perera, ed.: Plurilingüisme i educació: els reptes del segle XXI. Ensenyar llengües en la diversitat $i$ per a la diversitat, Barcelona, Institut de Ciències de l'Educació, Universitat de Barcelona, 373-380).

Pew Hispanic Center. <http://pewhispanic.org/>.

Potowski, Kim (2003): Programa y bibliografía del curso El español en los Estados Unidos (The University of Illinois at Chicago, EEUU). $<$ http://www.uic.edu/classes/span/span436/spaninus/index.html > [consulta: $18 / 05 / 2011]$.

Potowski, Kim (2004): Programa y bibliografía del curso La enseñanza del español a hispanohablantes (The University of Illinois at Chicago, EEUU). $\langle$ http://www.uic.edu/classes/span/span436/span436metodos/index.html > [consulta: 18/05/2011].

Potowski, Kim (2005): Fundamentos de la enseñanza del español a hispanohablantes en los EE.UU., Madrid, Arco Libros.

PotowsKI, Kim (2011): «La enseñanza del español a hispanohablantes en EEUU: Consideraciones pedagógicas y administrativas», Power Point disponible en: 
$<$ http://potowski.org/sites/potowski.org/files/Potowski_SNS_Cervates_NYC_March201 1.ppt>, y accesible desde la página 〈http://potowski.org/SNSresources> [consulta: $15 / 07 / 2011]$.

PotowsKi, Kim y Andrew LYNCH (2004): «La enseñanza del español en los Estados Unidos», Boletín de ASELE, 30, 23-40.

RAMOS, Jorge (2004): «Por qué los hispanos son distintos», en Vanguardia dossier. Los hispanos en Estados Unidos, n. ${ }^{\circ}$ 13, octubre/diciembre 2004, 35-48.

RocA, Ana y María Cecilia ColombI, eds. (2003): Mi lengua: Spanish as a heritage language in the United States, Washington, DC, Georgetown University Press.

RocA, Ana y María Cecilia COLOMBI (2003-2006): «Español para hispanohablantes: ¿Por qué iniciar y mantener un programa de español para hablantes nativos?», en Centro Virtual Cervantes, <http://cvc.cervantes.es/obref/espanol_eeuu/bilingue/aroca.htm> [consulta: 27/04/2011].

RUMBAUT, Rubén G. (2004): «Un retrato de la presencia hispana», en Vanguardia dossier. Los hispanos en Estados Unidos, n. ${ }^{\circ} 13$, octubre/diciembre 2004, 18-26.

Silva-CoRvalán, Carmen (2006): «Aspectos del desarrollo bilingüe españolinglés de niños en edad preescolar», en Mercedes Sedano, Adriana Bolívar y Martha Shiro, comp.: Haciendo Lingüística. Homenaje a Paola Bentivoglio, Caracas, Comisión de Estudios de Postgrado, Facultad de Humanidades y Educación, Universidad Central de Venezuela, 325-336.

SUÁREZ GARCíA, Jesús (2004): «Un caso de multiculturalidad y bilingüismo: la enseñanza del español a hijos de inmigrantes hispanos en Estados Unidos», en Manuel Pérez Gutiérrez y José Coloma Maestre, eds.: El español, lengua del mestizaje y la interculturalidad (Actas del XIII Congreso Internacional de la Asociación para la Enseñanza del Español como Lengua Extranjera, ASELE) (Murcia, 2-5 de octubre de 2002), 817-824. En Internet: <http://www.mepsyd.es/redele/biblioteca/asele/74.suarez.pdf> [consulta: 05/07/2011].

The National Foreign Language Center (NFLC) (Centro Nacional de Idiomas Extranjeros), proyecto REACH (Recursos para la Enseñanza y el Aprendizaje de las Culturas Hispanas). 〈http://www.nflc.org/REACH/>.

Torres TOrRes, Antonio (2001): «Culturas latinas en Estados Unidos», Cultura e Intercultura en la enseñanza del español como lengua extranjera, <http://www.ub.es/filhis/culturele/torres.html $>$.

TORRES TORRES, Antonio (2005): «El español en los Estados Unidos como expresión de identidades mixtas», en Emma Martinell Gifre y Erla Erlendsdóttir, eds., La conciencia lingüística europea: Nuevas aportaciones de impresiones de viajeros, Barcelona, PPU, 93-110. 
TORRES TORRES, Antonio (2010a): «El español de América en los Estados Unidos», en Milagros Aleza Izquierdo y José María Enguita Utrilla, coords.: La lengua española en América: normas y usos actuales, Valencia, Universitat de València. Con la colaboración de Marta Albelda Marco, Antonio Briz Gómez, Miguel Calderón Campos, Eduardo España Palop, Alejandro Fajardo Aguirre, Félix Fernández de Castro, David Giménez Folqués, Rosario Navarro Gala y Antonio Torres Torres, 403-427. Publicación en línea: 〈http://www.uv.es/aleza/esp.am.pdf>.

TORRES TORRES, Antonio (2010b): «Ejercicios sobre el español en los Estados Unidos y el spanglish», en Milagros Aleza Izquierdo y José María Enguita Utrilla. coords.: La lengua española en América: normas y usos actuales, Valencia, Universitat de València. Con la colaboración de Marta Albelda Marco, Antonio Briz Gómez, Miguel Calderón Campos, Eduardo España Palop, Alejandro Fajardo Aguirre, Félix Fernández de Castro, David Giménez Folqués, Rosario Navarro Gala y Antonio Torres Torres, 501-507. Publicación en línea: 〈http://www.uv.es/aleza/esp.am.pdf〉.

VALDÉs, Guadalupe M. (1995): «Bilingües y bilingüismo en los Estados Unidos: la política lingüística en una época antiinmigrante», Alteridades, 5 (10), 25-42. $\langle$ http://www.uam-antropologia.info/alteridades/alt10-2-valdes.pdf $>$ [consulta: 26/09/2010].

Zentella, Ana Celia et alii (1998): «Would you like your children to speak English and Spanish?» / «¿Quieren que sus hijos hablen el inglés y el español?» $<$ http://www.hunter.cuny.edu/blpr/bilingualism.html > [consulta: 20/06/2007]. 


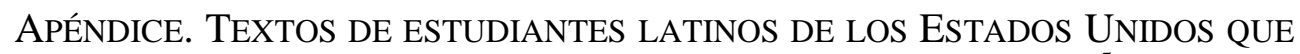 HAN SEGUIDO CURSOS DE ESPAÑOL EN LA UNIVERSIDAD DE BARCELONA ${ }^{5}$}

1. «iSon crueles las corridas de toros?»

«Mi familia es ranchera. Mi papa crecio en un rancho que tenia cosecha cada primavera y animales para críar. Cuando yo era niña todavia estaba vivo mi abuelo y el sigiya con la tradiccion de ser ranchero. Para la comida mis abuelos y tíos mataban los animales que tenian para comer. Entonces el acto de matar a un animal no lo pienso un acto violiento o cruel es algo que se tiene que ser para que come mi familia.

Es sierto que la corrida de toros es entretenimiento pero tambien lo ví como algo que se tuvo que ser para poder repartir la carne del toro. El matador se pone en mucho peligro sin algo para protejerse. Yo no creo que las corrídas de toros son crueles». (I. F., estudiante de California, de familia mexicana).

\section{A propósito del spanglish:}

«Considero que el spanglish es algo que se encuentra más frecuente en la segunda generación de estadounidenses. En mi caso yo llegué a los Estados Unidos a la edad de 10 años, ahora tengo 21 asi que más de la mitad de mi vida la he vivido en Estados Unidos. A mi el spanglish nunca se me dio mucho en parte porque es algo que a mi madre le molesta y constantemente me corrige cuando trato de usarlo. Mis hermanos pequeños quienes han crecido toda su vida en Estados Unidos se encuentran más atados al spanglish y lo utilizan constantemente.

Mis hermanos utilizan mucho las palabras "chirear" para referirce a "hacer trampa" usando como base la palabra "cheat" en inglés, y también usan mucho la palabra "chanza" o chance de "chance en inglés" para referirse a "oportunidad". Otras palabras de spanglish que se usan mucho en el sur de California entre los trabajadores del campo son las de "fil" para referirse a "campo" usando como base "field", "biles" para referirse a manufacturas usando como base "bill", "lonchear" "brekear" y "quitear" entre otras [...]. Algunas veces digo cosas como "Me haces fill out la aplicación" o "dile a tu hermano que lo vi de drug addict en down town"». (R. R., estudiante de California, de familia mexicana).

\footnotetext{
${ }^{5}$ La transcripción de los textos sigue con absoluta fidelidad el original. 


\section{A propósito del spanglish:}

«Cuando migran latinos a los Estados Unidos, y no aprenden inglés en escuela o no tienen ayuda o un profesor / tutor, a veces, se aprenden lentamente algunas palabras inglesas. Cuando hablan después de oir palabras, inyectan palabras convinientes, las que salen más facilmente, en sus frases. Por ejemplo mi abuelita de Panamá lleva cuarente y pico años en los estados unidos. Nunca ha aprendido inglés, pero yo no había aprendido el castellano cuando era joven, entonces, para comunicarnos empezaba a introducir palabras de inglés y español. Cuando jugábamos yo y mis primitos y los niños de la calle, en la calle nos decía, "DON' GO POR THAT WAY - ES DANGER PA'LLÁ!" De vez en cuando no aprenden mucho ingles los hijos de los inmigrantes, o a veces depende en que edad los traen los nos hacia los estados unidos. Aprenden los niños a escribir y entender inglés pero, hablar viene más difícilmente». (E. G., estudiante de California, de madre panameña).

\section{A propósito del spanglish:}

«Como hablante de este dialecto, el spanglish sí significa algo mucho más que un fenómeno lingüístico. Tal como lo identifican sus defensores, spanglish es una identidad que expone nuestro orgullo de ser una mezcla de dos culturas ya que es un lenguaje híbrido. Al hablar spanglish se hace más evidente nuestra presencia como una cultura en medio de dos culturas completamente distintas. Es una manera que encontrarnos e identificarnos en un país grandísimo, y así hacer una alianza. Además, es una manera de expresión creativa que está popularizándose [...]. Hoy ya no sólo es una manera de hablar, expresarse, identificarse, resistirse conscientemente, sino que es algo que toma vida inconscientemente también». (J. M., estudiante de origen mexicano).

\section{A propósito de The House on Mango Street, de Sandra Cisneros ${ }^{6}$ :}

«Otra cosa que resulta de grandes familias es que el padre esta ocupado trabajando para proveer para la familia, mientras la madre cuida de la casa y los niños más pequeños que resulta en algunos hijos no siendo educados apropiadamente. El mal cuidado de los hijos llega a ser tanto que a veces los niños hacen como quieren, como

${ }^{6}$ The House on Mango Street, que se publicó en 1984, es una de las obras más conocidas de la escritora chicana Sandra Cisneros. Refleja la vida cotidiana en un barrio latino de una gran ciudad de los Estados Unidos, a través del prisma de una niña llamada Esperanza. Sobre Sandra Cisneros y el lenguaje de su literatura, véase Antonio Torres (2009): «Heterogeneidad lingüística e identidad en la narrativa de Sandra Cisneros», Espéculo (Universidad Complutense de Madrid), 43. <http://www.ucm.es/info/especulo/numero43/sancisne.html>. 
los hijos de Rosa Vargas [personaje de la novela]. Ahora, regresando a lo de las viviendas siendo pequeñas y de condiciones muy malas hace que a gente como Esperanza soñar con una casa propia donde todo funcione.

A través de la cultura es como los hispanos se identifican, pero a la vez es su cultura la cual los lleva a la derrumba. En la cultura hispana hay roles muy distintos de géneros. El hombre es el proveedor y esta al frente de la casa, mientras la mujer es la ama de casa que se dedica a su hombre e hijos. Lo que recalca más la diferencia entere los sexos es el fenómeno del machismo. Donde el hombre es superior a la mujer y tiene todas las libertades de mundo mientras opresa a su esposa y la tiene atada al hogar.

Los asaltos sexuales y embarazos son cosas muy comunes dentro de la condición hispánica. Los chicanos o mexicano-estadounidense como Esperanza son bicultural. Por el lado anglo ven que la sexualidad es algo muy normal y por su lado mexicano ven que es algo malo, y como resultado de esto se sienten atrapados entre ambas culturas sin una guía. Los chicos tratan de enrollarse con cuantas chicas puedan, mientras las chicas quieren desarrollarse físicamente y ser sexy. A veces hay gente mayor, como el señor Benny que advierte a las chicas de andar usando los zapatos de tacón. Sin embargo la juventud no hace caso hasta que les pasa algo malo como un chico diciéndoles algo inapropiado o un borracho tratadles como prostitutas. Como punto final, esto se pudiera evitar se mejorar la comunicación entre padres e hijos». (A. J., estudiante de California, nacido en los Estados Unidos de padre y madre mexicanos).

\section{A propósito de The House on Mango Street, de Sandra Cisneros:}

«Nací en Puerto Rico y viví mis primeros dos años de vida allí con mi madre chilena y mi padre cubano. Es decir que mi lengua materna realmente es el español. Sin embargo, después de mudarnos a los EE.UU. y empezar en una escuela americana, empecé a favorecer el inglés. Ya no quería hablar en español en casa, y mis padres empezaron a hablarme en inglés. Esta situación continuó hasta el punto de que, cuando tenía 12 o 13 anos, no me gustaba identificarme con la población latina e intenté de esconder el patrimonio de yo y mis padres. En el libro, Esperanza refleja la misma actitud que yo tenía a esa edad.

Sin embargo, al entrar "high school", empecé a reconocer la importancia de mi patrimonio latino en mi vida. Recuerdo que tuve que enseñarme todas las conjugaciones verbales de nuevo, porque aunque siempre he podido entender el castellano, ya no era algo natural y fácil conjugar verbos. Pedí a mis padres que me hablaran en español, aunque el statu quo era hablarme en inglés. Ahora, mi identidad latina es algo importantísimo para mí, hasta el punto de que mi grupo musical favorito es Maná y mi actriz favorita es Penélope Cruz». (C. O., estudiante nacida en Puerto Rico de madre chilena y padre cubano). 
7. Sobre la propia experiencia migratoria:

«Puedo hablar de mi própria experiencia, siendo mexicana-americana. Nací en Estados Unidos pero al año me fui a vivir en México hasta que cumplí los siete. Para aquel entonces, el peso Mexicano había devalorado y básicamente eso había arruinado nuestro negocio allá. Vivíamos muy pobres, tanto que mi mamá no hallaba si usar los cinco pesos para comprar la leche del día o para comprar el billete del camión que me llevaba a mi escuela. Uno no sabe que haría o que desesperación sentiría hasta que esté en esa posición, viviendo en miseria. Lo que quiero decir es que cada inmigrante tiene su historia y propósito de salirse del país de origen. Llegando a Estados Unidos, tuve que aprender el ingles y al año lo capté. A partir de ahí, mi identidad se me desfiguró. En Estados Unidos, la asimilación ocurrió en forma natural con el tiempo pero nunca estuve de acuerdo con la forma en que me hacían identificarme con "mexicanoamericana" ya que en fin, eso no tiene real importancia. Es como ponerle mucho énfasis a las diferencias, que nos separa en lugar de concentrarnos en la idea de que estas diferencias son las que nos unen. Siempre hay que recordar que Estados Unidos es un país de inmigrantes. La identificación al final no importa pues ya se ha hecho todo el esfuerzo por adaptarme y asimilarme al país. La lucha de mis padres en querer mejorar la vida de nosotros aun sigue. Aun sigo peleando para mejorar la mía y la de mis padres, pero por eso no voy a dejar devaluar ni una ni otra parte de mi identidad. Sea lo que sea, quiero ser libre de expresarme y ser la persona bilingüe y bicultural que soy. Si trabajo y contribuyo al país y si así me respetan tal como soy, pues ¿qué más quiero? ¿Qué más quieren de mí? Creo que es una cooperación y entendimiento de ambas partes, el cual puede funcionar si se comunican y respetan como los seres humanos que somos». (J. N., estudiante de origen mexicano). 